\title{
Framework for Live Synchronization of RDF Views of Relational Data
}

\author{
Vânia M. P. Vidal ${ }^{\bowtie 1}$, Narciso Arruda ${ }^{1}$, Matheus $\mathrm{Cruz}^{1}$, Marco A. Casanova ${ }^{2}$, Valéria \\ M. Pequeno ${ }^{3}$ \\ ${ }^{1}$ Federal University of Ceará, Fortaleza, CE, Brazil \\ \{vvidal, narciso\}@lia.ufc.br, matheusmayron@gmail.com \\ ${ }^{2}$ Department of Informatics - Pontifical Catholic University of Rio de Janeiro, RJ, Brazil \\ casanova@inf.puc-rio.br \\ INESC-ID, Porto Salvo, Portugal \\ vmp@inesc-id.pt
}

\begin{abstract}
This Demo presents a framework for the live synchronization of an $\mathrm{RDF}$ view defined on top of relational database. In the proposed framework, rules are responsible for computing and publishing the changeset required for the RDB-RDF view to stay synchronized with the relational database. The computed changesets are then used for the incremental maintenance of the RDB_RDF views as well as application views. The Demo is based on the LinkedBrainz Live tool, developed to validate the proposed framework.
\end{abstract}

Keywords: RDF view $\cdot$ View Maintenance $\cdot$ Linked Data $\cdot$ Relational database.

\section{Introduction}

There is a vast content of structured data available on the Web of Data as Linked Open Data (LOD). In fact, a large number of LOD datasets are RDF views defined on top of relational databases, called $R D B-R D F$ views. The content of an RDB-RDF view can be materialized to improve query performance and data availability. However, to be useful, a materialized RDB-RDF view must be continuously maintained to reflect dynamic source updates.

Also, Linked Data applications can fully or partially replicate the contents of a materialized RDB-RDF view, by creating $R D F$ application views defined over the RDBRDF view. The generation of RDF application views improves the efficiency of applications that consume data from the LOD, and increases the flexibility of sharing information. However, the generation of RDF application views raises synchronization problems, since the original datasets can be continuously updated. Thus, updates on an RDB-RDF view must be propagated to maintain the RDF application views.

A popular strategy used by large LOD datasets to maintain RDF application views is to compute and publish changesets, which indicate the difference between two states of the dataset. Applications can then download the changesets and synchronize 
their local replicas. For instance, DBpedia (http://wiki.dbpedia.org) and LinkedGeoData (http://linkedgeodata.org/About) publish their changesets in a public folder.

In this demo, we show a framework, based on rules, that provides live synchronization of RDB_RDF views. In the proposed framework (see Fig. 1), rules are responsible for computing and publishing the changeset required for the RDB-RDF view to stay in synchronization with the relational database. The computed changesets are used by the synchronization tools for the incremental maintenance of RDB_RDF views and application views. In [8] we present a formal framework for automatically generating, based on the view mappings, the rules for computing correct changesets for an RDB-RDF view. Based on the mappings, at view definition time, we are able to: (i) identify all relations that are relevant for the view; and (ii) define the rules that compute the changeset required to maintain the view w.r.t an update over a relevant relation. Our formalism allows us to precisely justify that the rules generated by the proposed approach correctly compute the changeset. The demo video is available at http://tiny.cc/videolivesynrdbrdf (see also http://www.arida.ufc.br/livesynrdbrdf/).

The remainder of this paper is organized as follows. Section 2 describes our strategy, based on rules, for computing changesets for an RDB-RDF view. Section 3 summarizes related work. Section 4 covers an implementation and experiments. Section 5 presents the conclusions.

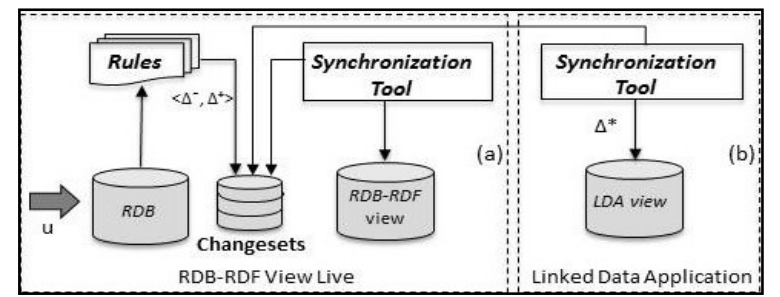

Fig. 1. Framework for live synchronization of RDB_RDF view.

\section{Computing Changesets for RDB-RDF Views}

In our strategy, we first have to identify the relations in $\boldsymbol{S}$ that are relevant for $\boldsymbol{V}$, that is, the relations whose updates might possibly affect the state of the view $\boldsymbol{V}$. For each such relation $R$, we define triggers that are fired immediately before and after an update on $R$, called before and after triggers, respectively, and which are such that:

BEFORE Trigger: computes $\Delta^{-}$the set of deleted triples

AFTER Trigger: computes $\Delta^{+}$the set of inserted triples.

The key idea of our strategy for computing the changesets is to re-materialize only the tuples whose RDF_State (the tuple triplification) might possibly be affected by the update. Thus, using $\Delta^{-}$and $\Delta^{+}$, one should be able to compute the new RDF state of the tuples that are relevant to the update (formal definitions in [7]). Fig. 2 shows the templates of the triggers associated with an update on a relation $R$.

For example, consider $u$, the UPDATE on R, where $r_{\text {old }}$ and $r_{\text {new }}$ are the old and new state of the updated tuple, respectively. Before the update, Trigger (a) is fired, and Procedure COMPUTE $\Delta_{-}[R]$ computes $\Delta^{-}$, which contains the OLD RDF_State of 
the tuples that are relevant to $\boldsymbol{V}$ w.r.t update $u$. After the update, Trigger (b) is fired. Using the database state after the update, Procedure COMPUTE $\Delta^{+}[R]$ computes $\Delta^{+}$, which contains the new RDF_State of the tuples that are relevant to $V$ w.r.t update $u$. Note that procedures COMPUTE $\Delta_{-}-[R]$ and COMPUTE $\Delta_{-} \Delta^{+}[R]$ are automatically generated, at view definition time, based on the view mappings [7]. Triggers for insertions and deletions are similarly defined and are omitted here.

Given $\mathrm{V}_{\mathrm{OLD}}$, the old state of the $R D B_{-} R D F$ view, in order to stay synchronized with the new state of database, the new state of the view is computed as $\mathrm{V}_{\mathrm{NEW}}=\left(\mathrm{V}_{\mathrm{OLD}}-\Delta^{-}\right) \cup \Delta^{+}$

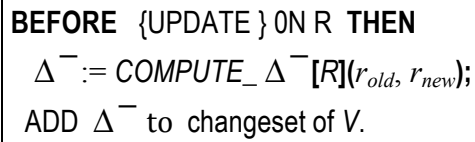

(a)

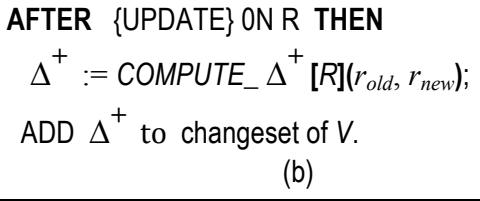

(b)

Fig. 2. Triggers to compute changeset of V w.r.t. updates on $R$

\section{Related Work}

The incremental view maintenance problem has been extensively studied in the literature for relational views [2], object-oriented views [6], semi-structured views [1], and XLM Views [3]. Despite their important contributions, none of these techniques can be directly applied to compute changesets for RDB-RDF views.

Comparatively less work addresses the problem of incremental maintenance of RDB-RDF views. Vidal et al. [8] proposed an incremental maintenance strategy, based on rules, for RDF views defined on top of relational data. Although the approach in this paper uses a similar formalism for specifying the view mappings, our strategy to compute changeset present in Section 2 differs considerably.

Faisal at al [4] presented an approach to deal with co-evolution, that is, the mutual propagation of the changes between a replica and its origin dataset. Their approach relies on the assumption that either the source dataset provides a tool to compute a changeset at real-time or third party tools can be used for this purpose. Thus, the contribution of this paper is complementary and relevant to satisfy their assumption.

Konstantinou et al [5] investigated the problem of the incremental generation and storage of the RDF graph that is the result of exporting relational database contents. In their approach, when one of the source tuples change, the whole triples map definition will be executed for all tuples in the affected table. By contrast, using our rules, we are able to identify which tuples are relevant to an update, and only the RDF_state of the relevant tuple are re-materialized.

\section{$4 \quad$ Implementation and Experiments}

To test our strategy, we implemented the LinkedBrainz Live tool ( $L B L$ tool), which propagates the upadates over the MusicBrainz database ( $M B D$ database) to the 
LinkedMusicBrainz view ( $L M B$ View). The $L M B$ View is intended to help $\mathrm{Mu}-$ sicBrainz (http://musicbrainz.org/doc/about) to publish its database as Linked Data. Figure 1 depicts the general architecture of our framework, based on rules, for providing live synchronization of RDB_RDF views. The main components of the $L B L$ tool are:

- Local MBD database: We installed a local copy of the $M B D$ database available on January 24, 2017.

- LMB View and Mappings: We created the R2RML mapping for translating $M B D$ data into the Music Ontology vocabulary (http://musicontology.com/), which is used for publishing the $L M B$ view. The $L M B$ view was materialized using the D2RQ tool (http://www.d2rq.org/). It took 67 minutes to materialize the view with approximately $41.1 \mathrm{~GB}$ of NTriples.

- Triggers: We reated the triggers to implement the rules required to compute and publish the changesets, as discussed in Section 2.

- LBL update extractor: This component extracts updates from the replication file provided by MusicBrainz, every hour, which contains a sequential list of the update instructions processed by the MusicBrainz database. When there is a new replication file, the updates should be extracted and then executed against the local database.

- LBL Syncronization tool: This component enables the LMB View to stay synchronized with the $M B D$ database. It simply downloads the changeset files sequentially, creates the appropriate INSERT/DELETE statement and executes it against the $L M B$ View triplestore.

In our experiments, we used the replication file with sequential number 101758 , which has 4,069 updates. Table 1 and Table 2 summarize our experimental results. Due to space limitation we consider only the relevant relations (RR) Artist and Track.

- Table 1 shows: The total number of tuples in the RR, the total time (in milliseconds) spent to triplify the RR, and the total number of updates on the RR.

- Table 2 shows: The average number of tuples relevant to updates on the RR, and the average time (in milliseconds) to compute the changeset $\left\langle\Delta^{-}, \Delta^{+}\right\rangle$for insertions (i) and updates $(\mathrm{u})$ on the relevant table. In the replication file, there is no deletion from the relations considered relevant.

The experiments demonstrated that the runtime for computing the changeset is negligible, when the number of relevant tuples is relatively small. This is what is expected, since the $R D B_{-} R D F$ View should be frequently updated to ensure that it remains consistent and up-to-date. We also analyzed the MusicBrainz replication file from one day and concluded that, in the experiments, just a small percentage of the tuples are relevant for the updates. Thus, we can conclude that the incremental strategy far outperforms full re-materialization, and also the re-materialization of the affected tables [5]. 
Table 1. Relevant relation Artist and Track

\begin{tabular}{|c|c|c|c|}
\hline $\begin{array}{c}\text { Relevant Relation } \\
\text { (RR) }\end{array}$ & $\begin{array}{c}\text { Number of } \\
\text { Tuple (k) }\end{array}$ & $\begin{array}{c}\text { Triplification } \\
\text { Time (ms) }\end{array}$ & $\begin{array}{c}\text { Number of } \\
\text { Updates }\end{array}$ \\
\hline Artist (a) & 1,166 & 340,721 & 40 \\
\hline Track (t) & 21,693 & 435,693 & 632 \\
\hline
\end{tabular}

Table 2. Changeset Computation Performance for RR Artist and Track

\begin{tabular}{|c|c|c|c|c|c|}
\hline Relevant Relation & \multirow{2}{*}{$\begin{array}{c}\text { Avg Number of } \\
\text { (RR) }\end{array}$} & \multicolumn{2}{|c|}{$\Delta^{-}$(avg time)(ms) } & \multicolumn{2}{|c|}{$\Delta^{+}$(avg time)(ms) } \\
\cline { 3 - 6 } & Relevant tuples & $\mathbf{i}$ & $\mathbf{u}$ & $\mathbf{i}$ & $\mathbf{u}$ \\
\hline Artist & 1.47 & 66 & 119 & 10 & 13 \\
\hline Track & 4.23 & 347 & 1531 & 154 & 993 \\
\hline
\end{tabular}

\section{Conclusions}

This Demo presented a framework for providing live synchronization of an RDF view defined on top of relational database. In the proposed framework, rules are responsible for computing and publishing the changeset required for the RDB-RDF view to stay synchronized with the relational database. The computed changesets are used for the incremental maintenance of the RDB_RDF views as well as application views.

We also implemented the LinkedBrainz Live tool to validate the proposed framework. We are currently working on the development of a tool to automate the generation of the rules for computing the changesets.

\section{References}

1. Abiteboul, S., McHugh, J., Rys, M., Vassalos, V., Wiener, J. L.: Incremental Maintenance for Materialized Views over Semistructured Data. In VLDB 1998, pp. 38-49 (1998)

2. Ceri, S. and Widom, J.: Deriving productions rules for incremental view maintenance. In VLDB 1991, pp. 577-589 (1991)

3. Dimitrova, K., El-Sayed, M., Rundensteiner, E.A.: Order-sensitive View Maintenance of Materialized XQuery Views. In ER 2003, pp. 144-157 (2003)

4. Faisal, S., Endris, K.M., Shekarpour, S., Auer, S.: Co-evolution of RDF Datasets. In: 16th International Conference - ICWE 2016 (2016)

5. Konstantinou, N., Spanos, D.E., Kouis, D., Mitrou, N.: An approach for the incremental export of relational databases into rdf graphs. International Journal on Artificial Intelligence Tools 24(2), (2015)

6. Kuno, H. A. and Rundensteiner, E. A.: Incremental Maintenance of Materialized ObjectOriented Views in MultiView: Strategies and Performance Evaluation. In IEEE TDKE, vol. 10, no. 5, pp. 768-792 (1998)

7. Vidal, V.M.P., Arruda, N., Casanova, M.A., Brito, C., Pequeno, V.M.: Computing Changesets for RDF Views of Relational Data. Technical Report, Federal University of Ceara (2017). Available at http://tiny.cc/TechnicalReportUFC2017

8. Vidal, V.M.P., Casanova, M.A., Cardoso, D.S.: Incremental Maintenance of RDF Views of Relational Data. In ODBASE 2013, pp 572-587 (2013) 